\title{
A lysosomal protease enters the death scene
}

\author{
Guy S. Salvesen
}

The Burnham Institute, 10901 North Torrey Pines Road, La Jolla, California 92037, USA. Phone: (858) 646-3114; Fax: (858) 646-3199; E-mail: gsalvesen@burnham.org.

The definition of lysosomes as cellular "suicide bags" derives from DeDuve's early work on fractionation and identification of cellular organelles. Since lysosomes contain degradative systems for proteins, carbohydrates, and lipids, it seemed natural that they should participate in cellular death and dismantling. But the last five years have seen a rapid advance in our understanding of the genetics and biochemistry of cell death, and it is apparent that a well conserved specific program exists in metazoans to execute superfluous or disobedient cells. This program is called apoptosis. Lysosomes or their constituents had not been considered to participate in this process under normal circumstances, and one cannot find discussion of these organelles or their constituents in the most recent series of eloquent reviews that cover the entire field of apoptosis (1-7). To be sure, there is evidence that lysosomal hydrolases participate in the unruly form of cell death known as necrosis and in the autophagic cell death found in neuronal populations (8). It is even evident that lysosomal rupture can trigger apoptosis under certain pathologic conditions (9), but to this point there has been little reason to believe that lysosomes participate in bona fide physiological apoptosis (Figure 1).

\section{Pathways to cell death}

The initiation and execution phases of apoptosis both require the activation of cysteine proteases of the caspase family. The executioner caspases 3 and 7 are responsible for the majority of limited proteolytic events that drive the protein machinery of the apoptotic response. These very specific proteases clip one or two bonds in proteins, either activating their substrates, as with certain apoptosis-related endonucleases and protein kinases, or inactivating them, as with transcription factors, mRNA splicing proteins, translation initiators, or cytoskeletal components. Together, these cleavages provide the biochemical signals required to kill and dismantle cells. There is currently no recognized role for noncaspase proteases in driving the execution phase. The initiation phase requires the activation of caspase 8 or caspase 9, which participate directly in the activation of the executioner zymogens. Interestingly, other proteases may also participate in the initiation phase, the most notable being the serine protease granzyme B (10).

Although the participation of other proteases in initiating apoptosis has often been proposed, no clear role for these enzymes has emerged. But now a lysosomal protease, cathepsin $\mathrm{B}$, has entered the fray. A recent paper in the

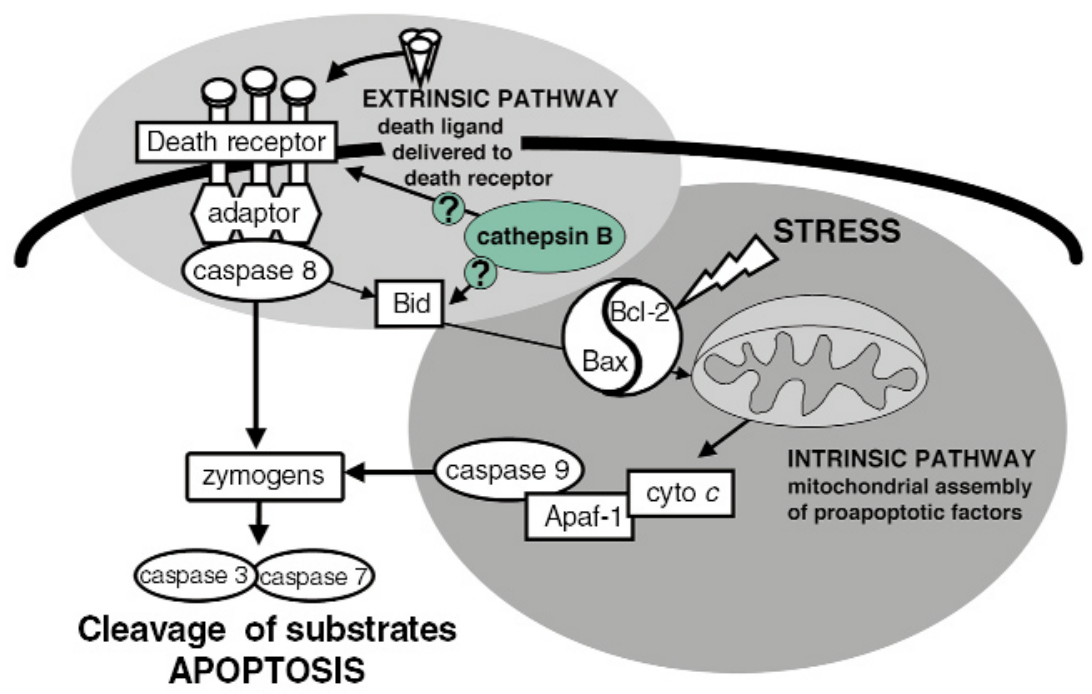

Figure 1

Two initiation pathways, triggered by separate events, converge to execute apoptosis. The extrinsic pathway (lighter shading) encompasses the extracellular ligation of death receptors by their cognate ligands, resulting in receptor clustering, adapter recruitment, and activation of the apical protease caspase 8. Thus, death receptors act as a conduit for the transmission of extracellular death signals into the cell's interior. The intrinsic pathway (darker shading) responds primarily to cellular stress (ionizing radiation, cytotoxic drugs, etc.), with the mitochondrion acting as an important integrator. Pro- and antiapoptotic members of the Bcl-2 family (Bax and $\mathrm{Bcl}-2$ serve as examples) regulate the lethal stress-response threshold. Activation of the apical protease caspase 9 occurs when it is driven into an active conformation by its cofactor Apaf- 1 , which itself requires prior binding to cytochrome $c$. Both pathways activate the executioner proteases caspases 3 and 7. The proapoptotic Bcl-2 family member Bid is at an intersection between the two initiation pathways. An important feature of the extrinsic pathway is that several receptors, in response to their cognate ligands, converge their death signals by activating caspase 8 . Now, new evidence (11) proposes a role for the lysosomal protease cathepsin B in the extrinsic pathway triggered by TNF- $\alpha$. 
between TNF- $\alpha$ treatment and the execution phase of apoptosis.

One reason not to have considered lysosomal cathepsins previously is that many of them - including cathepsin B are irreversibly inactivated in vitro at the $\mathrm{pH}$ found in the cytoplasm (12), whereas caspases are maximally active under these conditions (13). Nevertheless, placing cathepsin B just downstream of caspase 8 leads to a number of testable predictions, each descending from the current understanding of the varied signals emanating from death receptors. Since the most direct demonstration of a role for cathepsin B comes from the severely delayed apoptosis in the explanted hepatocytes of ablated mice, it is worthwhile to consider what other knockouts have told us of the relative importance of proteases in apoptosis.

\section{Phenotypes of caspase-deficient mice} Confirmation of the important roles of the caspases in apoptosis comes from gene ablation experiments in mice, in which the deficiency in apoptosis is very clear and leads to early embryonic lethality (caspase 8 ) or perinatal lethality (caspases 3 and 9) (14-16). Therefore, the otherwise normal development and phenotype of the cathepsin B knockout (17) seems initially to be at odds with the suggestion that cathepsin $\mathrm{B}$ is required for the extrinsic apoptosis pathway. If the cathepsin $\mathrm{B}$ apoptotic block is downstream of caspase 8 , then one would expect the same phenotype in the two knockout mice. This is not the case, since the caspase 8 knockout mouse is embryonically lethal, with death occurring around mid-gestation due to abnormal heart muscle development and erythrocytosis of major organs. Could cathepsin B be required for the Bid shunt from the extrinsic to the intrinsic pathway (Figure 1)? If this were the case, one would expect a phenotype similar to caspase 9 ablation. Apparently this is not the case, since caspase 9-/- mice display a developmentally impaired phenotype with delayed apoptosis and perinatal lethality.

One explanation for the block in apoptosis in explanted cathepsin $\mathrm{B}^{-/-}$ hepatocytes is that the effect may not be in the common areas of death signaling pathways, but more specifically related to the type 1 TNF receptor (TNFR-1, the main conduit for apoptotic signaling by TNF- $\alpha$ ). Mice ablated in this receptor have no obvious developmental phenotype, although they suffer from unresolved infectious diseases during adult life. TNFR-1 belongs to a family that includes Fas, the cognate receptor of Fas ligand (18). If cathepsin B is involved specifically in the TNF- $\alpha$ pathway, might the cathepsin B block also be seen following Fas ligation? If so, this would support the model proposed by Guicciardi et al. (11), which places the block downstream of caspase 8, a common position for both TNFR-1 and Fas.

Another possibility is that the apoptotic block reported for the cathepsin B knockout reflects changes in TNFR-1 protein levels. A substantial decrease in the receptor of cathepsin $B^{-/-}$mice may be an attractive explanation of the hepatocyte phenotype, given the purported role of this lysosomal protease in protein degradation. This could be checked by comparing steady-state levels of cell surface receptor in cathepsin $B^{-/-}$and cathepsin $B^{+/+}$hepatocytes. Moreover, if only the death pathway emanating from TNFR-1 is affected in the cathepsin B knockouts, then one would expect the competing rescue pathway transmitted by NF- $\kappa \mathrm{B}$ activation to be fully functional. Downregulation of the NF- $\mathrm{kB}$ pathway in cathepsin $B^{-/-}$hepatocytes would indicate decreased receptor protein levels or defects in the specific signaling assemblages of this multifunctional receptor, rather than a specific block of the apoptosis pathway.

In the latter scenario, the cathepsin B knockout would share features of the TNFR-1 knockout phenotype, raising another testable prediction. Inactivation of the NF- $\kappa$ B branch of TNF- $\alpha$ signaling, for example by ablation of RelA, is embryonically lethal due to sensitization to TNFR-1-mediated apoptosis. Because RelA protects the embryonic liver from TNFR-1-mediated apoptotic signals, this lethality can be rescued by crossing TNFR-1-ablated mice into the RelA ${ }^{--}$background (19). Will RelA-/lethality also be rescued in the cathepsin $B^{-/-}$background?

Irrespective of the location of the apparent block of TNF- $\alpha$ signaling in the absence of cathepsin B, the paper by Guicciardi et al. (11) opens up a fascinating biology for this much studied protease. It now joins its cousins cathepsins $\mathrm{L}$ and $\mathrm{S}-$ which participate in antigen processing for MHC-II loading (20, 21) - in mediating the response of cells to their environment. The lysosomal cysteine proteases can no longer be considered as simple garbage disposers.

1. Hengartner, M.O. 2000. The biochemistry of apoptosis. Nature. 407:770-776

2. Krammer, P.H. 2000. CD95's deadly mission in the immune system. Nature. 407:789-795.

3. Meier, P., Finch, A., and Evan, G. 2000. Apoptosis in development. Nature. 407:796-801.

4. Nicholson, D.W. 2000. From bench to clinic with apoptosis-based therapeutic agents. Nature. 407:810-816.

5. Rich, T., Allen, R.L., and Wyllie, A.H. 2000. Defying death after DNA damage. Nature. 407:777-783.

6. Savill, J., and Fadok, V. 2000. Corpse clearance defines the meaning of cell death. Nature. 407:784-788.

7. Yuan, J., and Yankner, B.A. 2000. Apoptosis in the nervous system. Nature. 407:802-809.

8. Kegel, K.B., et al. 2000. Huntingtin expression stimulates endosomal-lysosomal activity, endosome tubulation, and autophagy. J. Neurosci. 20:7268-7278.

9. Hill, I.E., Preston, E., Monette, R., and MacManus, J.P. 1997. A comparison of cathepsin B processing and distribution during neuronal death in rats following global ischemia or decapitation necrosis. Brain Res. 75:206-216.

10. Martin, S.J., et al. 1996. The cytotoxic cell protease granzyme B initiates apoptosis in a cell-free system by proteolytic processing and activation of the ICE,Ced3 family protease, CPP32, via a novel two-step mechanism. EMBOJ. 15:2407-2416.

11. Guicciardi, M.E., et al. 2000. Cathepsin B contributes to TNF- $\alpha$-mediated hepatocyte apoptosis by promoting mitochondrial release of cytochrome $c$. J. Clin. Invest. 106:1127-1137.

12. Turk, B., Turk, D., and Turk, V. 2000. Lysosomal cysteine proteases: more than scavengers. Biochim. Biophys. Acta.1477:98-111.

13. Stennicke, H.R., and Salvesen, G.S. 1997. Biochemical characteristics of caspases-3, $-6,-7$, and 8. J. Biol. Chem. 272:25719-25723.

14. Kuida, K., et al. 1998. Reduced apoptosis and cytochrome c-mediated caspase activation in mice lacking caspase 9. Cell. 94:325-337.

15. Kuida, K., et al. 1996. Decreased apoptosis in the brain and premature lethality in CPP32-deficient mice. Nature. 384:368-372.

16. Varfolomeev, E.E., et al. 1998. Targeted disruption of the mouse Caspase 8 gene ablates cell death induction by the TNF receptors, Fas/Apo1, and DR3 and is lethal prenatally. Immunity. 9:267-276.

17. Deussing, J., et al. 1998. Cathepsins B and D are dispensable for major histocompatibility complex class II-mediated antigen presentation. Proc. Natl. Acad. Sci. USA. 95:4516-4521.

18. Ashkenazi, A., and Dixit, V.M. 1998. Death receptors: signaling and modulation. Science. 281:1305-1308.

19. Rosenfeld, M.E., Prichard, L., Shiojiri, N., and Fausto, N. 2000. Prevention of hepatic apoptosis and embryonic lethality in RelA/TNFR-1 double knockout mice. Am. J. Pathol. 156:997-1007.

20. Nakagawa, T., et al. 1998. Cathepsin L: critical role in Ii degradation and CD4 T cell selection in the thymus. Science. 280:450-453.

21. Riese, R.J., et al. 1998. Cathepsin S activity regulates antigen presentation and immunity. J. Clin. Invest. 101:2351-2363. 